\title{
A New Feature-preserving Nonlinear Anisotropic Diffusion Method for Image Denoising
}

\author{
Zhen Qiu \\ zq15@hw.ac.uk \\ Lei Yang \\ trilithy@gmail.com \\ Weiping Lu \\ w.lu@hw.ac.uk
}

\author{
Department of Physics, \\ Heriot-Watt University, \\ Edinburgh, UK
}

\begin{abstract}
We present a new diffusion method for noise reduction and feature preservation. Presently, denoising methods commonly use a first-order derivative to detect edges in order to achieve a good balance between noise removal and feature preserving. However, if edges are partly lost to a certain extent or contaminated severely by noise, these methods may not be able to detect them and thus fail to preserve various features in images. To overcome this problem, we propose a new and more sophisticated feature detector by combining first- and second-order derivatives for a nonlinear anisotropic diffusion model. Numerical experiments show that the new diffusion filter outperforms many popular filters for denoising images containing edges, blobs and ridges and textures made of these features.
\end{abstract}

\section{Introduction}

Nonlinear diffusion is a popular denoising approach in which prior information of image features can be incorporated via a diffusion coefficient (DC) into the denoising processing. Methods based on nonlinear diffusion approaches have been applied for a variety of applications [1, 2, 3, 4]. In general, an edge is a fundamental feature that underlies more complicated features or structures in an image. The latter can be maintained as long as edges are preserved after a denoising process. Since edges can be characterised by a firstorder derivative (gradient), existing diffusion methods $[1,2,3,4]$ use the gradient as an edge detector to derive an appropriate coefficient that can reject diffusion at edges and permit smoothing in other places. This approach is simple but if edges are partly lost to a certain extent or are contaminated severely by noise, it may not be able to recover them and thus fail to preserve other features that are bounded by the edges.

A natural image usually consists of many features, such as edges, blobs, ridges, and textures that are made of these features, for example, striped and checkerboard-like patterns. Blobs and ridges are also fundamental features in images, which correspond to circular and line-like regions that are either brighter or darker than their surroundings respectively [9]. Mathematically, they are better described by a second-order derivative rather than a first-order which measures edges. In this paper we propose a novel feature- 


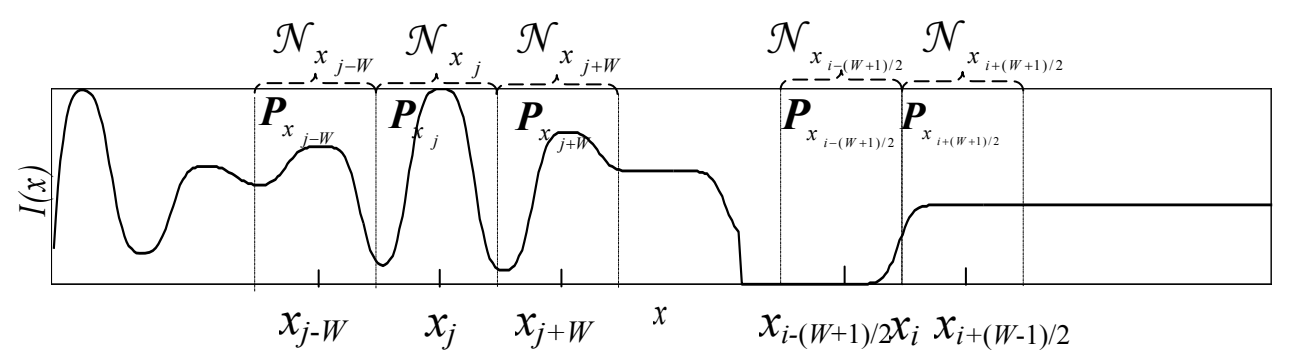

Figure 1: A schematic showing nonlocal difference in one-dimensional space

preserving denoising method by combining the first- and second-order nonlocal derivatives to form a new feature detector in a nonlinear diffusion model. By nonlocal derivative (NLD) we mean that the grayscale difference between two pixels is measured by two regions (patches) centred at the pixels. The use of nonlocal derivatives is inspired by the success of the popular nonlocal means (NLM) framework for image denoising $[5,6,7,8$, 11]. By combining the first- and second-order nonlocal derivatives, our new feature detector measures image intensity contrasts between neighbouring patches in a more sophisticated manner and can effectively capture fundamental features such as edges as well as blobs and ridges. We incorporate the new feature detector into a nonlinear diffusion model to form a new feature-preserving nonlinear anisotropic diffusion filter. Experimental results demonstrate that our denoising method can achieve a higher Peak-signal-to-noise ratio (PSNR) [6] and higher mean similarity index (MSSIM) [19] than several commonly used algorithms when applied to natural images containing a range of features and textures.

\section{Nonlocal Derivative}

\subsection{First-order Nonlocal Difference}

We describe the concept of the nonlocal difference in a one-dimensional (1-D) image. Extension to the two-dimensional (2-D) case is straightforward and will be discussed later. Let $I: \Omega \rightarrow \Re^{1}$ be a 1-D scalar image defined on the discrete domain $\Omega$ and $x \in \Omega$ is the pixel position, $x=x_{1}, x_{2}, \ldots, x_{N}$, as shown in Figure 1. For each pixel, $x$, we define a neighbourhood region, $\mathcal{N}_{x}$, which comprises $W$ pixels centred around $x$. We further define a patch $\boldsymbol{P}_{x}$, which is a vector comprising gray-level values of all pixels within the neighbourhood region $\mathcal{N}_{x}[10]$

$$
\boldsymbol{P}_{x}=\left[I\left(x_{-(W-1) / 2}\right), I\left(x_{-(W-1) / 2+1}\right), \ldots I(x), \ldots I\left(x_{(W-1) / 2}\right)\right]^{T},
$$

where $W$ is assumed to be an odd number for symmetry consideration. The nonlocal difference between two signal samples $I\left(x_{i}\right)$ and $I\left(x_{j}\right)$ can be measured as a Gaussian weighted Euclidean difference [5, 6, 10, 11, 13],

$$
d_{\mathrm{NL}}\left(I\left(x_{i}\right), I\left(x_{j}\right)\right)=\left\|\boldsymbol{P}_{x_{i}}-\boldsymbol{P}_{x_{j}}\right\|_{2, \sigma},
$$

of two vectors $\boldsymbol{P}_{x_{i}}$ and $\boldsymbol{P}_{x_{j}}$ in a $W$-dimensional space, where $\sigma$ is the standard deviation (Std) of a normalized Gaussian kernel. It is apparent that Eq. (2) involves a first-order difference between two vectors. For an image corrupted by noise, this nonlocal difference was considered to be a more reliable way to estimate $I\left(x_{i}\right)-I\left(x_{j}\right)$ from the noisy samples 
alone $[12,13]$, and was used in the NLM filter for improving denoising performance of traditional weighted averaging filters $[5,6,13]$.

From Eq. (2) we can define a first-order nonlocal derivative ( $\left.1^{\text {st }} \mathrm{NLD}\right)$ at pixel $x_{i}$ when $x_{j}$ approaches $x_{i}$,

$$
\left|\nabla_{\mathrm{NL}} I\left(x_{i}\right)\right|=\left\|\boldsymbol{P}_{x_{i-(W+1) / 2}}-\boldsymbol{P}_{x_{i(t(W+1) / 2}} \boldsymbol{Q}\right\|_{2, \sigma}=\left(\sum_{k=-(W-1) / 2}^{(W-1) / 2} G_{\sigma}(k)\left(I\left(x_{i-(W+1) / 2+k}\right)-I\left(x_{i+(W+1) / 2-k}\right)\right)^{2}\right)^{1 / 2},
$$

where $\boldsymbol{Q}=\left[\begin{array}{cccc}0 & \cdots & 0 & 1 \\ \vdots & 0 & 1 & 0 \\ 0 & \ddots & 0 & \vdots \\ 1 & 0 & \cdots & 0\end{array}\right]$ is a matrix with ones on the secondary diagonal and zeros elsewhere and $G_{\sigma}$ is a Gaussian kernel with Std $\sigma$. Eq. (3) involves the first-order difference between two adjacent patches $\boldsymbol{P}_{x_{i(W(1+1) / 2}}$ and $\boldsymbol{P}_{x_{i(t(1+1) / 2}}$, as depicted in Figure 1. For the same reason given to Eq. (2), Eq. (3) is more reliable than the pixel-level gradient operator involving two pixels to measure edges under noise contamination.

\subsection{Second-order Nonlocal Derivative}

Based on the definition of Eq. (3), we formulate a second-order nonlocal derivative $\left(2^{\text {nd }}\right.$ NLD)

$$
\begin{aligned}
\left|\nabla^{2}{ }_{\mathrm{NL}} I\left(x_{j}\right)\right| & =\left\|\boldsymbol{P}_{x_{j}}-\left(\boldsymbol{P}_{x_{j-W}}+\boldsymbol{P}_{x_{j+\psi}}\right) / 2\right\|_{2, \sigma} \\
& =\left(\sum_{k=-(W-1) / 2}^{(W-1) / 2} G_{\sigma}(k)\left(I\left(x_{j+k}\right)-\left(I\left(x_{j-W+k}\right)+I\left(x_{j+W+k}\right)\right) / 2\right)^{2}\right)^{1 / 2},
\end{aligned}
$$

which, as shown in Figure 1, involves a second-order difference between a central patch and two adjacent patches. A large $\left|\nabla^{2}{ }_{\mathrm{NL}} I\left(x_{j}\right)\right|$ corresponds to a brighter (darker) central patch compared with its neighbours, which indicates the presence of a blob (or ridge). For a similar reason given to Eq. (2), Eq. (4) can provide a more reliable measurement than a pixel-level second-order difference, i.e., the Laplacian operator [14], for blob and ridge detection in the presence of noise.

In general, responses of the $1^{\text {st }} \mathrm{NLD}$ and $2^{\text {nd }}$ NLD to edges and blobs are complex, but the expressions can be simplified in a special case where the patch size equals the blob size, i.e., $W=s$. We use this case as an example to explain the performance of the $1^{\text {st }}$ NLD and $2^{\text {nd }}$ NLD for detecting edge and blob features. Figure 2(a) draws a 1-D 8-bit image containing a step edge and a blob of size $s=21$ pixels without and with additive white Gaussian noise (AWGN) of a Std $\sigma_{\mathrm{n}}=40$. Intensities of the blob and edge are set to be 160 , against the backgrounds of 120. We apply Eq. (3) and Eq. (4) on the noise-free image and plot the responses in Figure 2(b), where the Std of the Gaussian function is set as $\sigma \rightarrow+\infty$ for simplicity. As seen from this figure, the response of $1^{\text {st }}$ NLD to the edge and that of $2^{\text {nd }}$ NLD to the blob are both uminodal and symmetric. For the step edge the peak values of the $1^{\text {st }}$ NLD is twice higher than that of the $2^{\text {nd }}$ NLD, whereas for the blob the two derivatives are the same. However, when the image is contaminated with noise, for the blob the peak values of the $2^{\text {nd }}$ NLD is higher than that of the $1^{\text {st }}$ NLD, as shown in Figure 2(c). This is because $2^{\text {nd }}$ NLD measures the difference of two neighbouring $1^{\text {st }}$ NLDs. When one of the two $1^{\text {st }}$ NLDs fails to detect one edge of the blob due to noise 


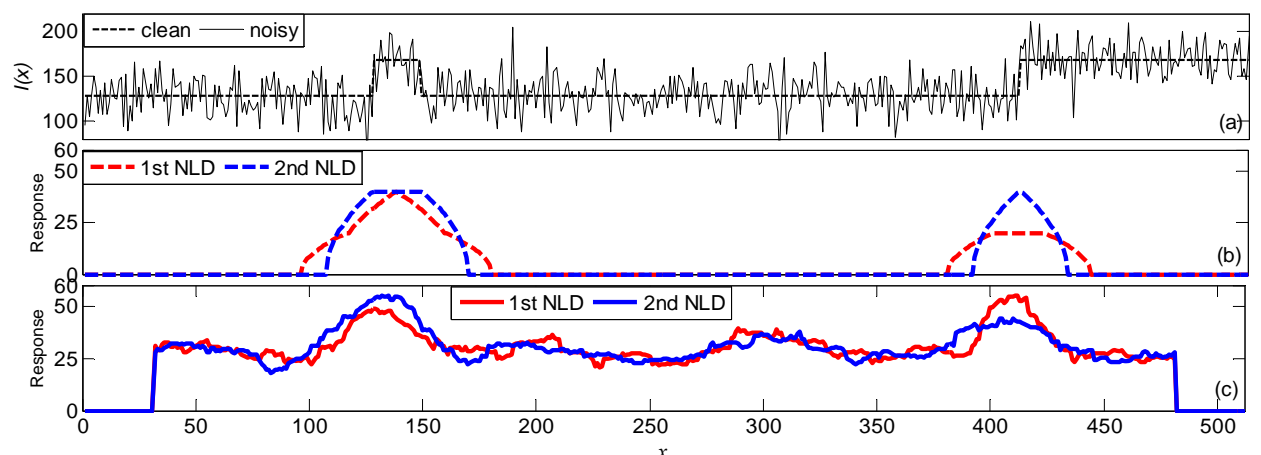

Figure 2: (a) A 1-D noise-free image containing one blob and one step edge and the corresponding image corrupted by additive white Gaussian noise with a Std $\sigma_{\mathrm{n}}=40$; (b) The $1^{\text {st }}$ NLD and $2^{\text {nd }}$ NLD of the noise-free image; (c) The $1^{\text {st }}$ NLD and $2^{\text {nd }}$ NLD of the noisy image

contamination, the $2^{\text {nd }}$ NLD can still give a reasonable response if the other edge of the blob can be detected. Therefore, the $2^{\text {nd }}$ NLD is a more robust blob detector compared to the edge detector $\left(1^{\text {st }} \mathrm{NLD}\right)$. Finally we note that in the general cases of $W \neq s$, the essential characteristics of the $1^{\text {st }}$ NLD and $2^{\text {nd }}$ NLD for edge and blob detection remains unchanged.

\section{Feature-Preserving Nonlinear Diffusion}

In this section, we exploit a new feature detector that combines first- and second-order nonlocal derivatives to form a more sophisticated feature-preserving nonlinear diffusion (FP-ND) compared to the traditional nonlinear diffusion model [1],

$$
\frac{\partial I\left(x_{i}, t\right)}{\partial t}=\operatorname{div}\left[c\left(\left|\nabla_{\mathrm{NL}, \sigma} I\left(x_{i}, t\right), \nabla_{\mathrm{NL}, \sigma}^{2} I\left(x_{i}, t\right)\right|\right) \cdot \nabla I\left(x_{i}, t\right)\right],
$$

where the diffusion coefficient (DC)

$$
c\left(\left|\nabla_{\mathrm{NL}, \sigma}^{2} I\left(x_{i}, t\right)\right|\right)=\exp \left[-\left(\left(w_{1} \cdot\left|\nabla_{\mathrm{NL}, \sigma} I\left(x_{i}, t\right)\right|+w_{2} \cdot\left|\nabla_{\mathrm{NL}, \sigma}^{2} I\left(x_{i}, t\right)\right|\right) / h\right)^{2}\right],
$$

$I\left(x_{i}, t=0\right)=I_{0}\left(x_{i}\right)$ is the initial noisy image, $\nabla$ is the gradient operator and div is the divergence operator. The DC in Eq. (6) is a decreasing function of the two detectors. The weights $w_{1}$ and $w_{2}$ should be appropriately chosen for balancing the contributions of $1^{\text {st }}$ NLD and $2^{\text {nd }}$ NLD. We define $w_{1}$ and $w_{2}$ as,

$$
w_{1}=\frac{\left|\nabla_{\mathrm{NL}, \sigma} I\left(x_{i}, t\right)\right|}{\left|\nabla_{\mathrm{NL}, \sigma} I\left(x_{i}, t\right)\right|+\left|\nabla_{\mathrm{NL}, \sigma}^{2} I\left(x_{i}, t\right)\right|} \text { and } w_{2}=\frac{\left|\nabla^{2}{ }_{\mathrm{NL}, \sigma} I\left(x_{i}, t\right)\right|}{\left|\nabla_{\mathrm{NL}, \sigma} I\left(x_{i}, t\right)\right|+\left|\nabla^{2}{ }_{\mathrm{NL}, \sigma} I\left(x_{i}, t\right)\right|} \text {. }
$$

In the vicinity of an edge, $\left|\nabla_{\mathrm{NL}, \sigma} I\left(x_{i}, t\right)\right|>\left|\nabla^{2}{ }_{\mathrm{NL}, \sigma} I\left(x_{i}, t\right)\right|$, so $w_{1}>w_{2}$ and the $1^{\text {st }}$ NLD contributes more to the DC; in the vicinity of a blob, $\left|\nabla_{\mathrm{NL}, \sigma} I\left(x_{i}, t\right)\right|<\left|\nabla_{\mathrm{NL}, \sigma}^{2} I\left(x_{i}, t\right)\right|$, therefore $w_{1}<w_{2}$ and the $2^{\text {nd }}$ NLD contributes more. Since the DCs are small in the vicinity of both features and high in other regions, the diffusion (smoothing) process will be discouraged considerably in feature regions and encouraged in background regions, leading to a feature-preserving nonlinear diffusion (FP-ND) method that preserves features and removes noise in the backgrounds. 
The threshold $h$ is a parameter that determines whether a feature should be preserved in the diffusion process. A large $h$ may oversmooth features whereas a small $h$ can produce artefacts and unsatisfactory noise suppression. The choice of $h$ should also reflect noise levels. We employ median absolute deviation (MAD) of $w_{1} \cdot\left|\nabla_{\mathrm{NL}, \sigma} I\left(x_{i}, t\right)\right|+w_{2} \cdot\left|\nabla_{\mathrm{NL}, \sigma}^{2} I\left(x_{i}, t\right)\right|$ for a robust estimation of the diffusion threshold $h[3,6]$.

A simple way to terminate the diffusion process is by fixing the number of iterations. In this work we utilize the mean absolute error (MAE) criterion [16] to stop the diffusion adaptively. The diffusion process is ceased only when the MAE reaches to a pre-specified small value.

\section{Experiments}

In this section we present visual and numerical results obtained by using our diffusion method, first for a 1-D image and subsequently 2-D images. In the latter case we incorporate the orientation of the features into Eq. (5), leading to a FP-nonlinear anisotropic diffusion (FP-NAD) model. We test the FP-NAD on 2-D natural images and compare the results with existing popular denoising methods, including PM anisotropic diffusion method [1], structure adaptive filter (SAFIR) [6] and block matching and 3-D collaborative filtering (BM3D) [8]. The last method is considered to be the best denoising algorithm at present $[11,12,13]$. We have not included bilateral filter [25], which can be seen as a special case of SAFIR [6], because reports $[6,26]$ have already shown that it underperforms SAFIR for image denoising.

\subsection{1-D Images}

We first test the FP-ND filter Eq. (5) on the 1-D noisy image of Figure 2(a). The patch size $W$ is chosen as $W=21$. The initial value of the diffusion threshold $h$ is set to be $h=40$, same as the Std $\sigma_{\mathrm{n}}$ of the AWGN in the image, and is updated using the MAD operator at each iteration. We first consider a special case for $w_{1}=1$ and $w_{2}=0$, for which the FP-ND Eq. (5) is reduced to a conventional edge-preserving diffusion. Figure 3(a) shows the results by this diffusion method in which the step edge is preserved but the blob is smoothed out. On the contrary, if we set $w_{1}=0$ and $w_{2}=1$, the FP-ND Eq. (5) is reduced to a blob-preserving diffusion model. Figure 3(b) shows results by this method in which

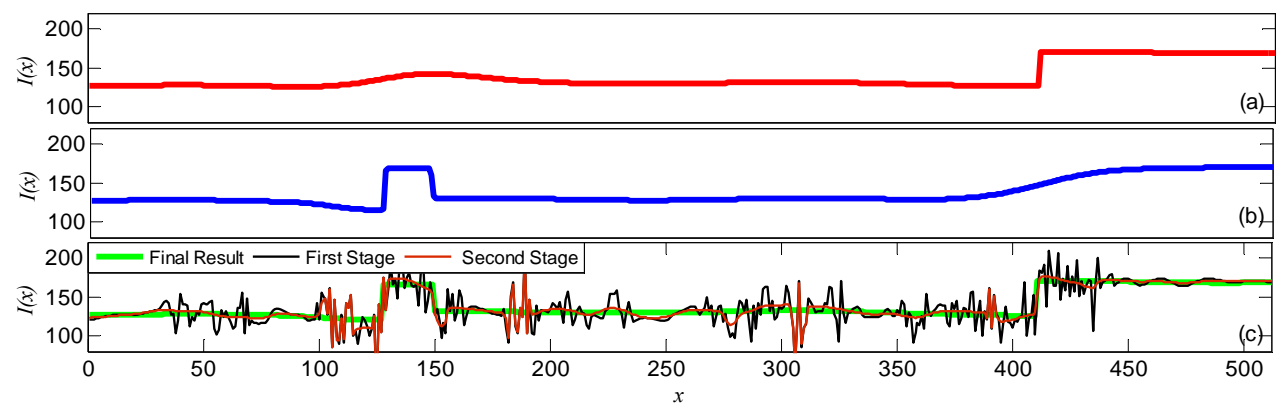

Figure 3: Tests on the noisy image shown in Figure 2(a). (a) Denoising result by an edgepreserving diffusion, which is a special case of the FP-ND when $w_{1}=1$ and $w_{2}=0$; (b) Denoising result by a blob-preserving diffusion, which is also a special case of the FP-ND when $w_{1}=0$ and $w_{2}=1$; (c) Denoising result by the FP-ND, where $w_{1}$ and $w_{2}$ is calculated by Eq. (7). 
the blob is preserved but the step edge filtered.

We now apply the FP-NAD to the noisy image and show the denoising results in three different stages during the diffusion process in Figure 3(c). When $w_{1}$ and $w_{2}$ follow Eq. (7), the $1^{\text {st }}$ and $2^{\text {nd }}$ NLD play a dominating role around edges and blobs respectively in determining the DCs. As a result, for the initial noise image, DCs are low in the vicinity of both features and high in backgrounds. As such, FP-ND smoothes more heavily on the former regions in the initial stage while leaves the regions in the vicinity of the edge and blob features essentially unchanged, as shown by the black curve in Figure 3(c). As the image evolves during the diffusion process, the smoothing effect "propagates" towards the feature regions. Background regions away from the features continue to be smoothed during this period. The contrasts of the features thus become increasingly higher, giving rise to higher responses of the $1^{\text {st }}$ and $2^{\text {nd }}$ NLD around the edge and blob, respectively. Higher responses of $1^{\text {st }}$ and $2^{\text {nd }}$ NLD imply higher $w_{1}$ and $w_{2}$ respectively, so the system performs in a positive feedback manner, leading to more effective noise reduction and feature preservation in the second stage, as shown in Figure 3(c) (red curve). As noise is gradually removed, the difference of the images between two adjacent iterations becomes increasingly smaller. The diffusion process stops when the MAE is reduced to 0.01. As seen from Figure 3(c), the final result shows good preservation of features and reduction of noise compared to the noise-free image in Figure 2(a).

\subsection{2-D Images}

In 2-D images, edges and ridges can have different orientations and blobs can be directional ellipses. To better preserve the geometric properties of these features, the orientations of features should be taken into account when we apply the FP-ND filter to 2$\mathrm{D}$ images. We therefore propose a spatially anisotropic nonlinear diffusion, namely diffusion along rather than across the principal direction of the features [17]. In this case, the scalar DC $c$ in Eq. (5) should be replaced by a $2 \times 2$ diffusion tensor (DT) $D$, a symmetric and definite-positive matrix [18],

$$
\frac{\partial I\left(\boldsymbol{x}_{i}, t\right)}{\partial t}=\operatorname{div}\left[\boldsymbol{D} \nabla I\left(\boldsymbol{x}_{i}, t\right)\right],
$$

where $\boldsymbol{x}_{i}=\left[x_{i}, y_{i}\right]$ is a pixel in 2-D images and $\nabla I\left(\boldsymbol{x}_{i}, t\right)=\left[\nabla_{x} I\left(\boldsymbol{x}_{i}, t\right), \nabla_{y} I\left(\boldsymbol{x}_{i}, t\right)\right]^{T}$ is a vector whose elements are gradients at the pixel $\boldsymbol{x}_{i}$ along the $x$-axis and $y$-axis. The DT $\boldsymbol{D}$ is constructed to have the same eigenvectors $\left(\boldsymbol{V}_{0}, \boldsymbol{V}_{1}\right)$ as a structure tensor $J=\left(\nabla G_{\sigma} * I\right)\left(\nabla G_{\sigma} * I\right)^{T}[17]$, implying that the diffusion flux $\boldsymbol{D} \nabla I$ is decomposed onto two orthonormal basis with directions across and along the principal direction of features, respectively. Eigenvalues of $\boldsymbol{D}$ are

$$
\lambda_{0}\left(x_{i}, y_{i}, t\right)=c\left(\left|\nabla_{N L, \sigma} I\left(x_{i}, y_{i}, t\right)\right|,\left|\nabla_{N L, \sigma}^{2} I\left(x_{i}, y_{i}, t\right)\right|\right) \text { and } \lambda_{1}\left(x_{i}, y_{i}, t\right)=\sqrt{\lambda_{0}\left(x_{i}, y_{i}, t\right)},
$$

where $c\left(\left|\nabla_{N L, \sigma} I\left(x_{i}, y_{i}, t\right)\right|,\left|\nabla_{N L, \sigma}^{2} I\left(x_{i}, y_{i}, t\right)\right|\right)$ is given by the DC Eq. (6) except that the $1^{\text {st }}$ and $2^{\text {nd }}$ NLD is now in the 2-D form of

$$
\begin{aligned}
& \left|\nabla_{N L, \sigma} I\left(x_{i}, y_{i}, t\right)\right|=\sqrt{\left\|\boldsymbol{P}_{x_{i}, y_{i}}-\boldsymbol{P}_{x_{i-k}, y_{i}} \boldsymbol{Q}\right\|_{2, \sigma}^{2}+\left\|\boldsymbol{P}_{x_{i}, y_{i}}-\boldsymbol{P}_{x_{i}, y_{i-\mu}} \boldsymbol{Q}\right\|_{2, \sigma}^{2}}
\end{aligned}
$$

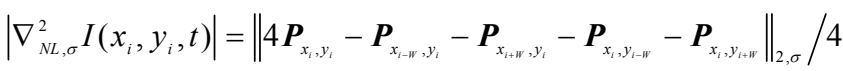


The reason for designing Eq. (9) follows the explanation in [18]: In background regions Eq. (8) performs an isotropic smoothing due to $\lambda_{0} \approx \lambda_{1} \rightarrow 1$ and in the vicinity of features Eq. (8) performs an anisotropic smoothing along the principal direction of features due to $\lambda_{0}$

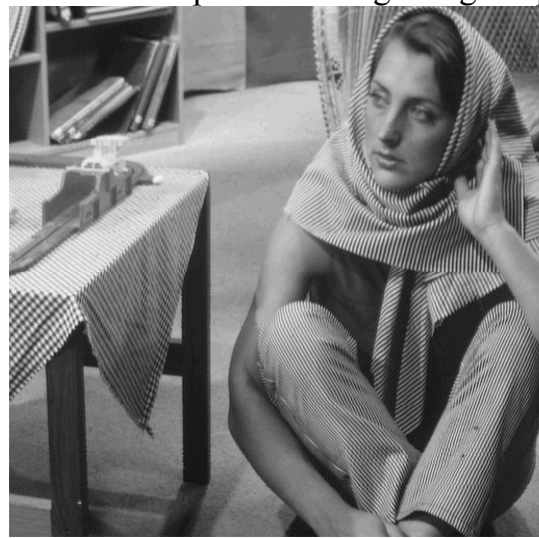

(a)

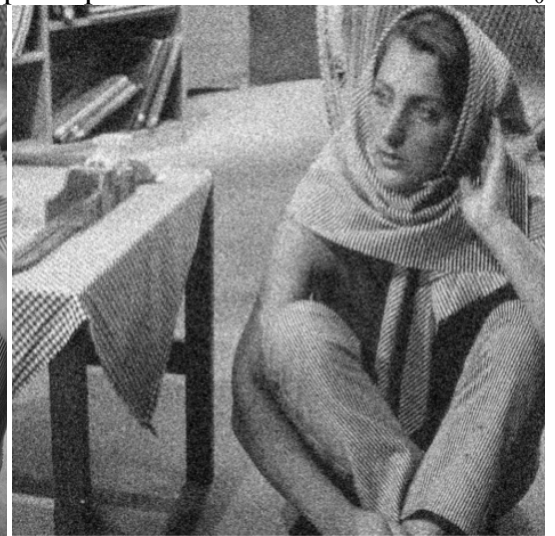

(b)

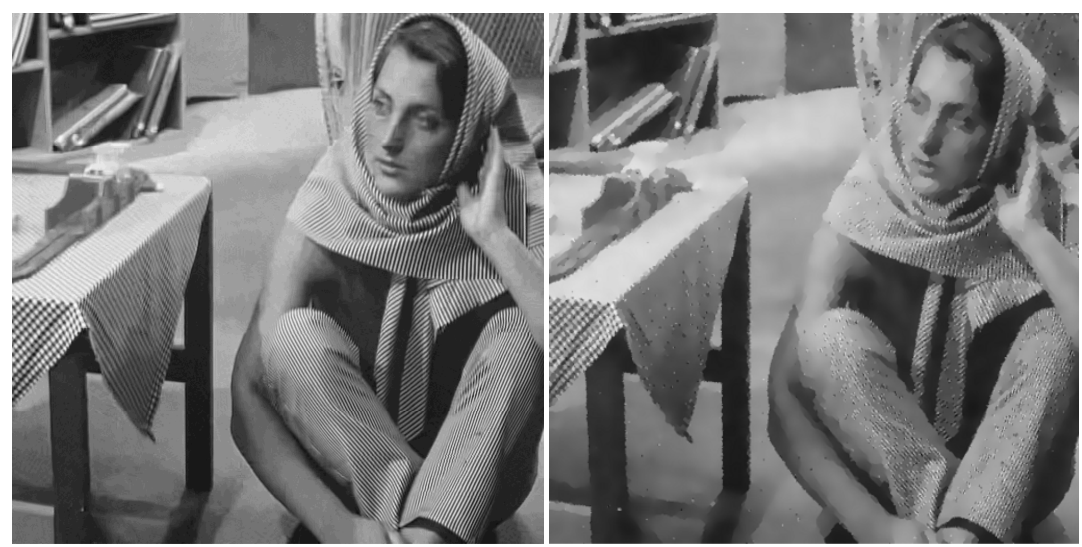

(c)

(d)

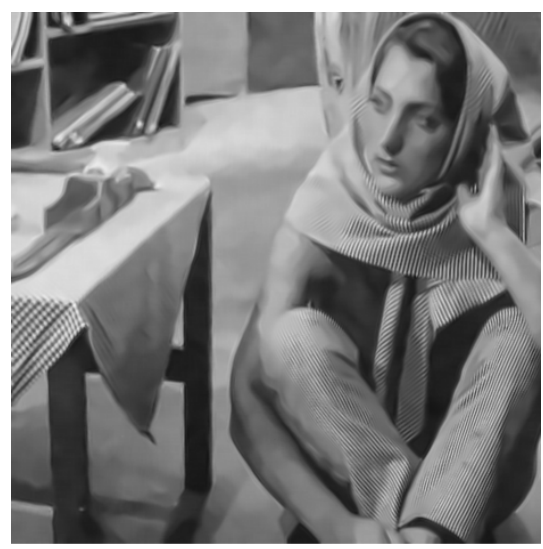

(e)

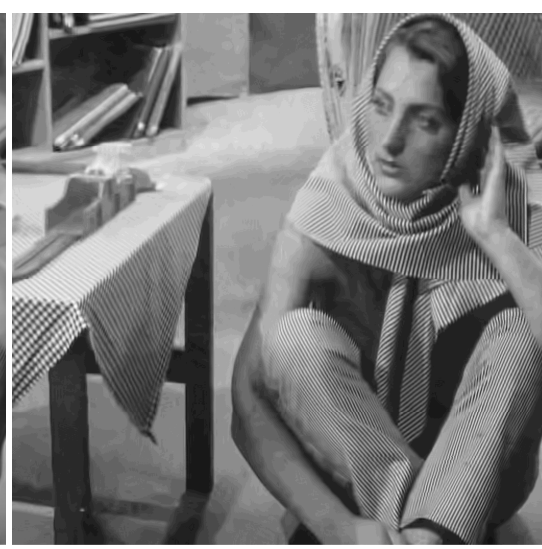

(f)

Figure 4: (a) A noise-free Barbara image; (b) The noisy image with AWGN of a Std $\sigma_{\mathrm{n}}=25$; (c) -(f) Denoised results by FP-NAD, PM,SAFIR and BM3D, respectively. 
$<\lambda_{1} \rightarrow 0$.

\subsubsection{Denoising of a Natural Image Containing Multiple Features}

We first undertake experiments on a classical image: Barbara (512x512). Figure 4(a) and (b) shows the noisy-free and noisy image with AWGN of a Std $\sigma_{\mathrm{n}}=25$. As seen, the image contains various features, including many edges, checkerboard-like and striped textures on the tablecloth and striped textures on the clothes.

We apply the feature-preserving nonlinear anisotropic diffusion (FP-NAD) filter Eq. (8) to the noisy image Figure 4(b). The patch size for calculating $1^{\text {st }}$ NLD by Eq. (3) and $2^{\text {nd }}$ NLD by Eq. (4) is set to be $13 \times 13$ pixels, which is between the smallest and largest widths (7 to 17 pixels) of the ridges in the image. The parameter $h$ is chosen initially to be $h=25$, equal to the Std $\sigma_{\mathrm{n}}$ of the noise, and is updated using the MAD operator at successive iterations. The diffusion process stops when the MAE is less than 0.01. The denoised result by our FP-NAD is shown in Figure 4(c). As seen, all features in the image are correctly preserved by comparing to the noise-free image Figure 4(a), including eyes of Barbara, weak striped textures on the clothes of Barbara and checkerboard-like textures on the tablecloth.

The denoised results of the same image by PM, SAFIR and BM3D, are shown in Figure 4(d)-(f). For PM, the time interval is set to be $\Delta t=0.2$ and the processes stop when the MAE is less than 0.01. In SAFIR, the patch window is set to be $9 \times 9$, the maximum number of increments for the nested window size is 4 , the critical parameters $\lambda_{0.01}=113.5$ and $\rho=3$. The reasons for choosing these parameter values are explained in an original paper [6]. The parameters for BM3D used in all tests follow 'Normal Profile' in Table I in reference [8].

\begin{tabular}{|c|c|c|c|c|c|}
\hline \multirow{2}{*}{$\sigma_{\mathrm{n}}$} & \multicolumn{5}{|c|}{ PSNR/MSSIM values } \\
\cline { 2 - 6 } & Noisy Image & FP-NAD & PM & SAFIR & BM3D \\
\hline \hline 25 & $20.32 / 0.406$ & $\mathbf{3 1 . 2 2 / 0 . 9 0 1}$ & $24.47 / 0.710$ & $27.78 / 0.790$ & $30.73 / 0.887$ \\
30 & $18.79 / 0.346$ & $\mathbf{3 0 . 3 7 / 0 . 8 9 2}$ & $24.03 / 0.635$ & $26.39 / 0.748$ & $29.76 / 0.864$ \\
40 & $16.49 / 0.264$ & $\mathbf{2 8 . 8 5 / 0 . 8 4 3}$ & $22.16 / 0.514$ & $24.30 / 0.674$ & $28.07 / 0.824$ \\
\hline
\end{tabular}

Table 1: Comparison of PSNR and MSSIM by our method, PM, SAFIR and BM3D. Three levels of AWGN with Stds $\sigma_{\mathrm{n}}=25,30$ and 40 are tested.

By a visual comparison, PM oversmoothes the eyes and almost wipes out the weak striped patterns on the tablecloth and trousers. SAFIR preserves the features on the trousers better than the PM, but still oversmoothes the eyes of Barbara and the stripes on the tablecloth. Our FP-NAD avoids this problem since we combine different feature detectors together to provide high responses on these features under noise contamination. The strong feature preserving ability of our method is also attributed to the unimodal shape of the $1^{\text {st }}$ and $2^{\text {nd }}$ NLD and anisotropic diffusion along the orientation of the features. Moreover, our method performs isotropic diffusion in background regions so remove noise and induce little artefacts, unlike the PM method. BM3D performs comparably with our FP-NAD in terms of feature preserving, but tends to have lower image contrast of the eyes and stripes on the trousers by comparison. We have quantified the image fidelity by calculating peak signal-to-noise ratio (PSNR) [6] and mean structure similarity index (MSSIM) [19] between original and denoised images. Higher PSNR and MSSIM imply better image restoration and structure preservation, respectively. We report in Table 1 the PSNR and MSSIM values of denoised results shown in Figure 4 by our method, PM, SAFIR and 


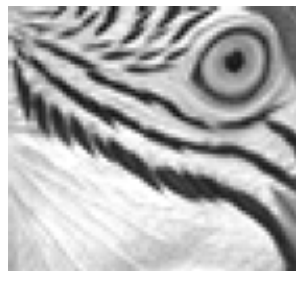

(a) $+\infty / 1$

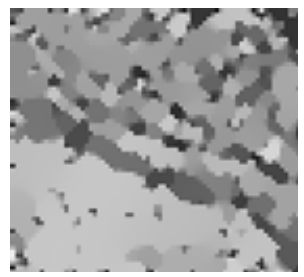

(d) $13.43 / 0.378$

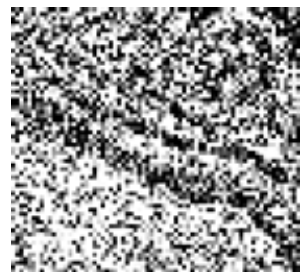

(b) $8.40 / 0.223$

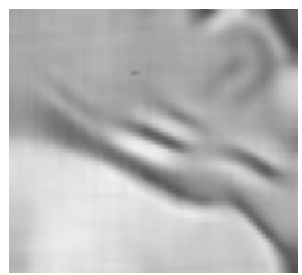

(e) $14.68 / 0.361$

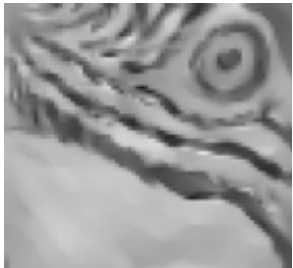

(c) $15.29 / 0.622$

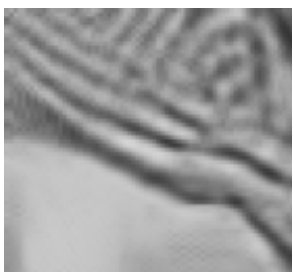

(f) $14.46 / 0.484$

Figure 5. Test on a fragment of a natural image, Parrots. (a) Noise-free image; (b) Noisy image $\left(\sigma_{\mathrm{n}}=120\right)$; (c) - (f) Denoised results by our method, PM, SAFIR and BM3D, respectively. Two numbers under each image are the corresponding PSNR and MSSIM values.

BM3D. Table 1 also summarizes the PSNR and MSSIM for the denoised images by the above methods on Figure 4(a) with AWGN of Stds $\sigma_{\mathrm{n}}=30$ and 40. As seen, our method, for different levels of AWGN, achieves the highest PSNR and MSSIM value among the four algorithms.

\subsubsection{Denoising of a Natural Image under Extremely Severe Noise Contamination}

We further test the FP-NAD filter on a natural image under severe noise contamination. Figure 5(a) and (b) show a fragment of a noise-free and noisy benchmark image Parrots [20], which contain a blob (eye of the parrot) and several ridges (eye socket of the parrot and dark stripes on its face) of different sizes and orientations. Extremely high-level AWGN $\left(\sigma_{\mathrm{n}}=120\right)$ is used in order to test the performance limit of the FP-NAD filter on low-PSNR images. Parameters of our filter, PM, SAFIR and BM3D are the same as those in the last test. The denoising result is shown in Figure 5(c) - (f), the two numbers under each figure are the corresponding PSNR and MSSIM values, respectively. As seen, due to the severity of noise, edges in the image Figure 5(b) are heavily broken, particularly in the eye region. As such, edge-preserving diffusion, such as PM (Figure 5(d)), is ineffective in restoring these features. PM also generates artefacts in flat regions. Visually, SAFIR and BM3D are shown to preserve features better than PM, but are still outperformed by the FP$\mathrm{NAD}$, as the eye, eye socket and face stripes are partly oversmoothed in comparison to the result by FP-NAD. The main reason behind the good performance of our FP-NAD is again due to the combination of two feature detectors. Moreover, nonlinear anisotropic diffusion employed in our filter has the ability to effectively reconstruct the shapes of the features while remove noise.

\section{Conclusions and Discussion}


We have presented a new feature-preserving nonlinear anisotropic diffusion method in which the diffusion coefficient is constructed by a combination of different feature detectors. We have tested the new algorithm on 1-D and 2-D images and demonstrated good performance in preserving edges, blobs, ridges and textures. It can also effectively reduce the background noise and create minimal artefacts.

A key issue in our FP-NAD filter is the formation of DC by using a combination of $1^{\text {st }}$ NLD and $2^{\text {nd }}$ NLD, which provides improved detection performance on edge, blob and ridge features. The NAD process controlled by this DC can therefore smooth out noise while preserve features. We note that our work provides a general diffusion framework under which multiple feature detectors can be combined into the diffusion model. A range of choices of these detectors are already available in the fields of image processing and computer vision $[15,21,22,23,24]$. A future direction of our work is therefore to study a more comprehensive set of feature detection operators in the feature-preserving denoising for a wide range of applications. The work will also focus on optimizing system parameters such as the patch size $W$ and the iteration number in order to maximize the performance of the FP-NAD.

\section{References}

[1] P. Perona and J. Malik. Scale-space and edge detection using anisotropic diffusion. IEEE Transactions on Pattern Analysis and Machine Intelligence, 12(7): 629-639, 1990.

[2] L. Rudin, S. Osher, and E. Fatemi.. Nonlinear total variation based noise removal algorithms. Physica D, 60: 259-268, 1992.

[3] M. Black., G. Sapiro, D. Marimont and D. Heeger. Robust anisotropic diffusion. IEEE Transactions on Image Processing, 7(3): 421-432, 1998.

[4] C. Barcelos, M. Boaventura and E. Silva. A well-balanced flow equation for noise removal and edge detection. IEEE Transactions on Image Processing, 12(7): 751-763, 2003.

[5] A. Buades, B. Coll, and J. M. Morel. A review of image denoising algorithms, with a new one. SIAM Multiscale Modeling and Simulation, 4(2): 490-530, 2005.

[6] C. Kervrann and J. Boulanger. Optimal spatial adaptation for patch-based image denoising. IEEE Transactions on Image Processing, 15(10): 2866-2878, 2006.

[7] M. Elad and M. Aharon. Image denoising via sparse and redundant representations over learned dictionaries. IEEE Transactions on Image Processing, 15(12): 3736-3745, 2006.

[8] K. Dabov, A. Foi, V. Katkovnik and K. Egiazarian. Image denoising by sparse 3-D transformdomain collaborative filtering. IEEE Transactions on Image Processing, 16(8): 2080-2095, 2007.

[9] Wikipedia. http://en.wikipedia.org/wiki/Blob_detection.

[10] D. Tschumperlé and L. Brun. Non-local image smoothing by applying anisotropic diffusion PDE's in the space of patches. In Proceedings of IEEE International Conference on Image Processing, 2957-2960, 2009.

[11] P. Chatterjee and P. Milanfar. Is denoising dead? IEEE Transactions on Image Processing, 19(4): 895-911, 2010.

[12] V. Katkovnik, A. Foi, K. Egiazarian and J. Astola. From local kernel to nonlocal multiplemodel image denoising. International Journal of Computer Vision, 86(1): 1-32, 2010. 
[13] L. Pizarro, P. Mrázek, S. Didas, S. Grewenig and J. Weickert. Generalised nonlocal image smoothing. International Journal of Computer Vision, 90(1): 62-87, 2010.

[14] R. Gonzalez and R. E. Woods. Digital image processing, $2^{\text {nd }}$ edition. Prentice Hall, 2002.

[15] M. Jacob and M. Unser. Design of steerable filters for feature detection using canny-like criteria. IEEE Transactions on Pattern Analysis and Machine Intelligence, 26(8): 1007-1019, 2004

[16] F. Zhang, Y. M. Yoo and L. M. Koh.. Nonlinear diffusion in Laplacian pyramid domain for ultrasonic speckle reduction. IEEE Transactions on Medical Imaging, 26(2): 200-211, 2007.

[17] J. Weickert. Coherence-enhancing diffusion filtering. International Journal of Computer Vision, 31(2-3): 111-127, 1999.

[18] D. Tschumperlé and R. Deriche. Vector-valued image regularization with pde's: A common framework for different applications. IEEE Transactions on Pattern Analysis and Machine Intelligence, 27(4): 506-517, 2005.

[19] Z. Wang, A.C. Bovik, H.R. Sheikh and E. P. Simoncelli. Image quality assessment: From error visibility to structural similarity. IEEE Transactions on Image Processing, 13(4): 600-612, 2004

[20] http://www.r0k.us/graphics/kodak/kodim23.html.

[21] D. G. Lowe. Distinctive image features from scale-invariant keypoints. International Journal of Computer Vision, 60(2): 91-110, 2004.

[22] R. Unnikrishnan, C. Pantofaru and M. Hebert. Toward objective evaluation of image segmentation algorithms. IEEE Transactions on Pattern Analysis and Machine Intelligence, 29(6): 929-944, 2007.

[23] E. Rosten, R. Porter and T. Drummond. Faster and Better: A machine learning approach to corner detection. IEEE Transactions on Pattern Analysis and Machine Intelligence, 32(1): $105-119,2010$.

[24] O. Tuzel, F. Porikli and P. Meer. Pedestrian detection via classification on riemannian manifolds. IEEE Transactions on Pattern Analysis and Machine Intelligence, 30(10): 1713$1727,2010$.

[25] C. Tomasi and R. Manduch. Bilateral filtering for gray and color images. In Proceedings of International Conference on Computer Vision, 839- 846, 1998.

[26] M. Zhang and B. Gunturk. Multiresolution bilateral filtering for image denoising. IEEE Transactions on Image Processing, 17(12): 2324-2333, 2004. 